\title{
SOME OBSERVATIONS ON VENOUS PRESSURE ESTIMATIONS IN THE LOWER LIMB
}

\author{
A. M. Boyd, B. N. Catchpole, R. P. Jepson and S. S. Rose, Manchester, England
}

From the Professorial Surgical Unit, Manchester Royal Infirmary

In this paper we report an investigation of venous pressures during exercise in ambulant patients. The investigation was undertaken because we wished to assess more accurately the abnormal venous mechanisms in patients complaining of swollen, painful or ulcerated legs. Phlebography, upon which great reliance has formerly been placed, has two limitations. Firstly, it yields only a knowledge of anatomical or architectural venous defects, which may be difficult or impossible to correlate with the accompanying symptoms. Secondly, phlebograms are difficult to interpret and may give misleading information. The method described below has enabled us to obtain functional as well as anatomical data; and from a correlation of the two, a better understanding of many lower leg syndromes has been gained.

With the advent of major surgical procedures for the treatment of post-phlebitic limbs, such as popliteal and femoral vein ligation, it has become more than ever necessary to have reliable objective criteria by which to assess the results of treatment. Rest in bed, healing of the ulcer, adequate bandaging and support, and psychological factors can easily cloud the issue, especially when the follow-up period is a matter of a few months or years. Since an abnormal venous pressure is the most important factor in the causation of symptoms in the post-phlebitic limb, readings taken before and after operation enable the efficacy of the treatment to be judged more accurately.

\section{METHOD}

The indirect methods of measuring venous pressure (Beecher et al. 1936) are inaccurate in legs with thick indurated skin, and impossible if much oedema is present. Moreover they do not allow the final phlebogram to be performed.

The apparatus that we use (Fig. 1 ) is a modification of Walker's. A length of polythene tubing with an internal bore of half or one millimetre is introduced through a small incision under local anaesthesia into a superficial dorsal vein of the foot. The vein is tied off distally. Through the tubing is passed normal saline solution from an ordinary transfusion bottle connected with a mercury manometer and bulb. The saline pressure necessary to hold the column of advancing blood stationary in the polythene tubing is taken as the intravenous pressure. If blood does not run immediately into the polythene tube when the transfusing pressure is lowered sufficiently the tube should be gently withdrawn or pushed slightly farther up the vein, as its tip may lie behind a valve cusp. Blood must not be allowed to reach the needle joining the polythene to the rubber transfusing tube because if it does so it may clot; it may even clot within the polythene tube if kept still for too long. It should be remembered that saline is being injected into the patient by a positive air pressure, and precautions must be taken to replenish the saline as necessary.

With the apparatus thus set up, the patient is asked to stand erect and relaxed. After a minute or two, when the pressure has become stable, measurements may be begun. These are made by lowering the pressure in the apparatus until blood appears in the polythene tube, where it is kept stationary or, during exercise, with a small range of oscillation. The manometer reading is then noted. (The actual pressure at the tip of the polythene tube, with the patient and saline bottle both standing on the ground, is slightly different from the manometer reading. It is equivalent to the sum of the pressures of the column of mercury in the manometer and that due to the height of saline in the reservoir, less that of a column of blood from ground level to the tip of the polythene tube.) The apparatus may be modified 
by including a water manometer connected via a three-way tap to the polythene tube. By joining the polythene tube directly to the water manometer, the column of saline which the renous pressure will sustain can be directly noted against the patient's body.

Routine measurements - Four routine readings are taken, all with the patient in the erect position, namely: 1) The resting venous pressure (r.v.p.); 2) the venous pressure with the patient " marking time" by taking about forty six-inch-high steps a minute-the exercise renous pressure (e.v.p.); 3) and 4) the pressures as in 1) and 2) with an Esmarch rubber bandage applied from ankle to knee.

Continuous recordings-We have also obtained continuous records of venous pressure variations in the exercising leg. A short length of capillary tube, with a trumpet-shaped end

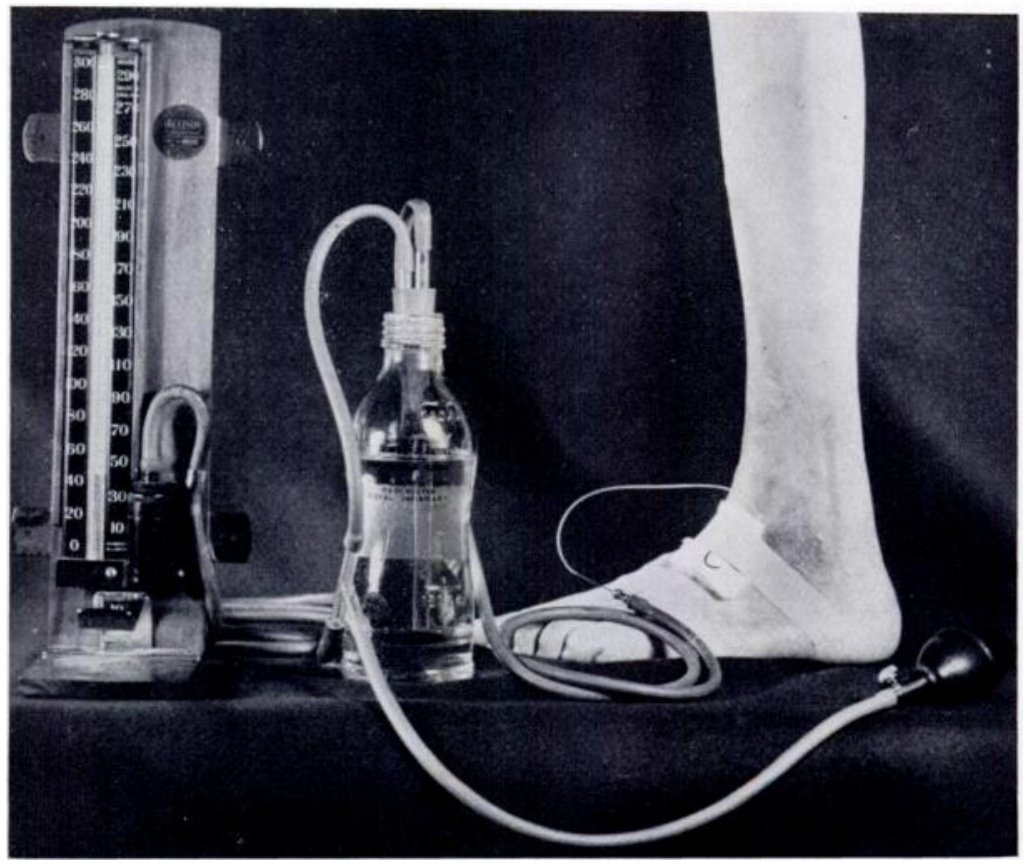

FIG. 1

The apparatus used for measuring venous pressure in the lower limb.

over which a tight rubber diaphragm is stretched, is coupled directly with the polythene tube entering the vein. On the diaphragm rests a very light coil moving frcely over an iron core. By suitable apparatus, the changes in electrical inductance of the moving coil can be made to produce a direct current, which will give a tracing on a Hughes recorder. Thus pressure variations moving the diaphragm and coil can be continuously recorded. The patient stands on a small platform with a contact arranged to mark the tracing while weight is borne on the leg under examination. A time signal is also incorporated. This apparatus has enabled us to observe the time relationships of pressure variations with exercise. Furthermore, the speed with which the pressure falls during exercise and returns to its resting level can be accurately measured.

Phlebograms-After the pressure readings have been completed phlebograms can be obtained via the polythene tube.

Care of the leg-The apparatus is simple to take down; the polythene tube is withdrawn, the ligatures cut out and a haemostatic pressure dressing applied, to be removed in four or five days. With careful aseptic technique the tiny incision heals by first intention. These investigations can be repeated on the same leg, even elderly patients generally raising no objection. The method is quite suitable for out-patients. 


\section{VENOUS PRESSURES IN NORMAL LIMBS}

For the assessment of the range of normal pressures, we have investigated the lower limb of healthy volunteers, mostly young adults. The symptomless limbs of patients, many of whom had symptoms in the other leg, have served as controls in the middle-aged and elderly. The relationship of pressures between a superficial dorsal vein of the foot and the deep calf veins is not known, but from the limited studies we have made we believe that the two run parallel, for the rich intramuscular and intermuscular network and the numerous communicating veins between the deep collecting and the superficial systems allow rapid equalisation of pressure.

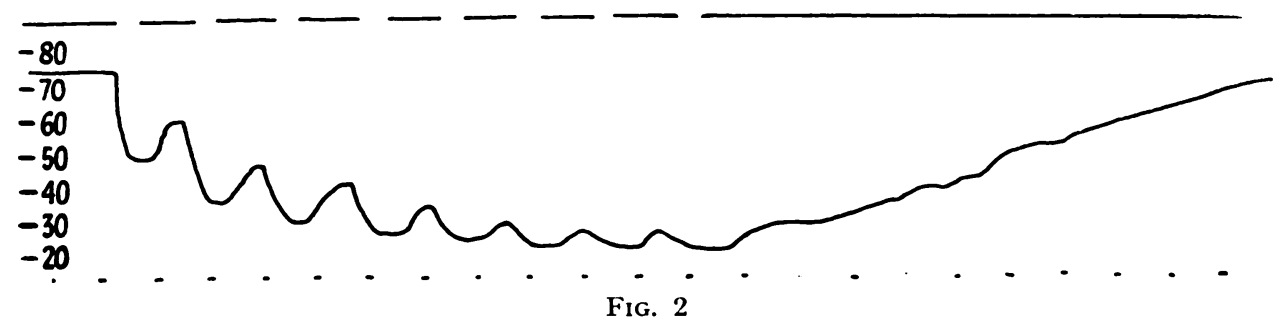

A continuous record of venous pressure during exercise of a normal lower limb. (The highest trace breaks when the leg under examination leaves the ground. The lowest trace marks the time in seconds. The scale is in millimetres of mercury.)

Standing venous pressure-This approximates to a saline column the height of the manubrium sterni. Mercury manometer readings often showed a slightly lower pressure, but when more precise readings with the fine saline manometer were taken the second intercostal space was usually reached. In the occasional case, however, the standing vertical pressure corresponded to a level below that of the manubrium sterni.

Exercise pressures in the normal leg-The fall of pressure in the superficial dorsal vein immediately exercise is begun is rapid and maintained. It can be studied best by the use of the continuous recording apparatus (Fig. 2).

The first tonic thrust of the leg muscles may raise the pressure slightly and a fall does not occur until the foot is lifted from the ground and relaxation of the lower leg muscles has occurred. As can be seen from the tracings the pressure then falls by many millimetres of mercury and if the tonic and relaxation phases are repeated two or three times a plateau level below 60 per cent of the pre-exercise reading is reached. Figure 3 shows the range of percentage falls in exercise venous pressure in a series of patients grouped according to age and sex. Rapid and persistent exercise may cause a further fall and, when measured by the mercury manometer, the readings during exercise may approach zero; continuous and energetic exercise for many minutes in volunteers failed to raise the pressure. The pressures recorded from both lower limbs were alike, never differing by more than 5 millimetres of mercury. The size of polythene tubing or of the dorsal vein did not significantly alter the readings obtained.

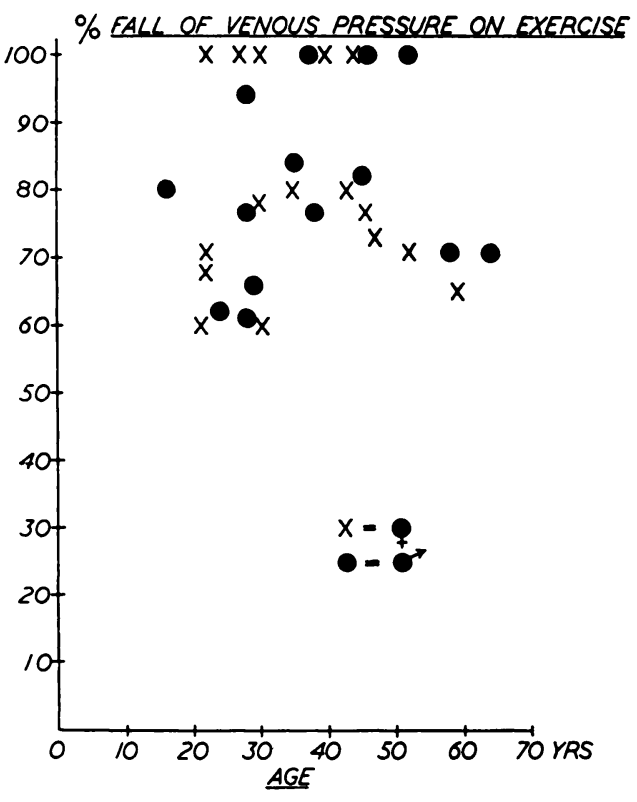

Fig. 3

A graph of percentage falls of venous pressure on exercise of the normal limb plotted against the patient's age.

vol. 34 B, No. 4, NOVEMBER 1952 


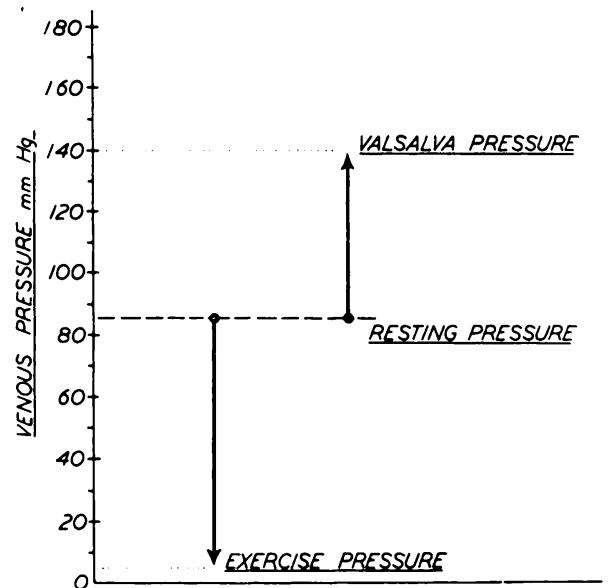

FIG. 4

1)iagram illustrating the effects of exercise and a Valsalva test on venous pressure measured in a superficial dorsal vein of the foot in a normal lower limb.

All routine measurements of pressure were repeated after the firm application of an Esmarch rubber bandage from the knee to the ankle. In normal subjects the pressure readings remained within a few millimetres of those before the bandaging. The effect of a Valsalva test (a forced expiratory effort against a closed glottis) on the normal limb is shown in Figure 4. Some factors in the venous return from the lower limb-Figures 5 and 6 show phlebograms of a normal leg. The opaque fluid was injected into a superficial dorsal vein of the foot with the volunteer standing erect and relaxed. It will be seen that five minutes later the fluid, whose specific gravity is approximately that of blood, has remained almost stationary. This agrees with the finding of Smirk that the volume of the limb increases perceptibly on standing; and indeed, if the pressure in the deep veins corresponds with that recorded in the dorsal foot veins, it is difficult to understand how an adequate venous return from the lower leg is maintained during the erect relaxed position. In practice the position of standing relaxed without movement of the calf is most unusual

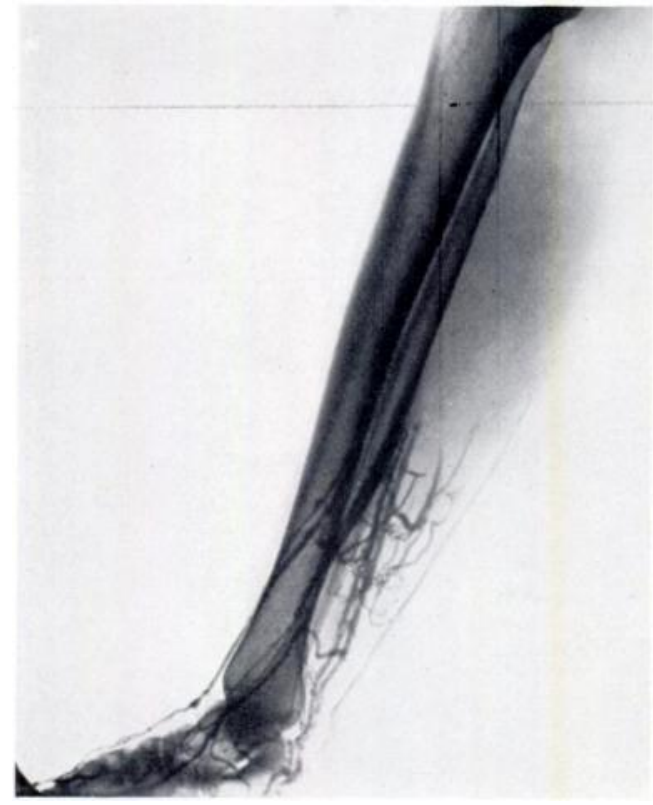

Fig. 5

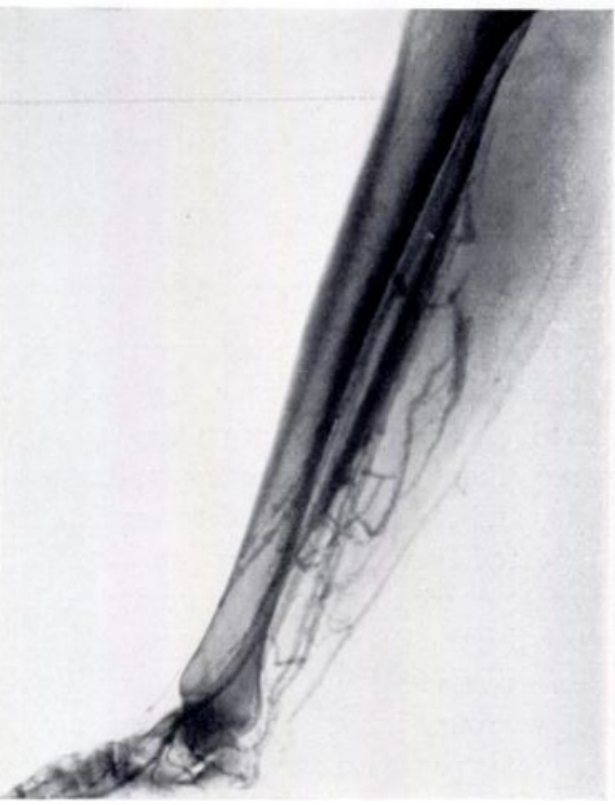

Fig. 6

Figure 5-Phlebogram of a normal leg immediately after injection of 20 cubic centimetres of 50 per cent diodone into a superficial dorsal vein of the foot. Figure 6-The leg has been kept perfectly still; five minutes later a second phlebogram shows that the diodone has flowed upwards very little.

and needs considerable concentration to maintain it. What normally happens is that the subject moves constantly, now relaxing now contracting the calf muscles. Each small movement causes some return of blood by mechanical compression. When blood reaches the abdomen further factors such as the diaphragmatic pump aid its return. 
The anatomy of the lower limb is admirable for the promotion of venous return by exercise. The thick muscles of the calf and anterior compartment are tightly ensheathed in fascia; when the long-bellied muscles contract, their thickest part moves proximally, as is seen readily in the gastrocnemius muscle when the patient stands on tip-toe. This movement massages blood up the deep collecting veins. Furthermore, the rich valvular arrangement in the deep intermuscular veins compels flow in an upward direction. During the relaxation phase the valveless intramuscular veins fill quickly, like a sponge, and the intermuscular veins receive blood from the superficial system as the deep venous pressure falls. The popliteal vein acts mainly as a conduit from the "tibial " to the "femoral " venous pump. High venous pressure may be caused by a defect in either, for instance by tenotomy of the tendo calcaneus, which weakens the calf muscle (Fig. 7), or by ankylosis of the knee,

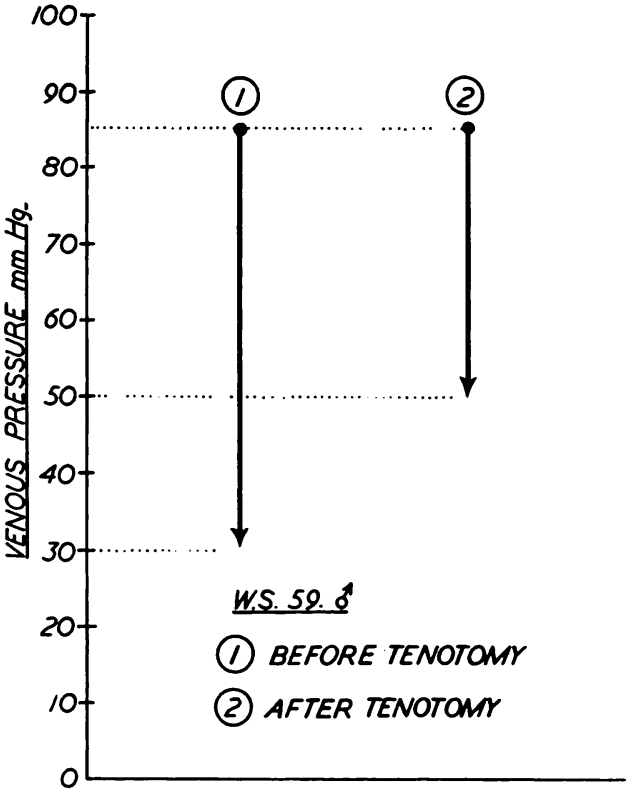

Fig. 7

The effects of tenotomy of the tendo calcaneus on venous pressure measured in the foot. which weakens the quadriceps.

Pressure readings in other veins-It is difficult to obtain volunteers for venous pressure readings in the major limb veins. Nevertheless standing and exercise pressures were obtained from the common femoral, common iliac and inferior vena cava in three patients.

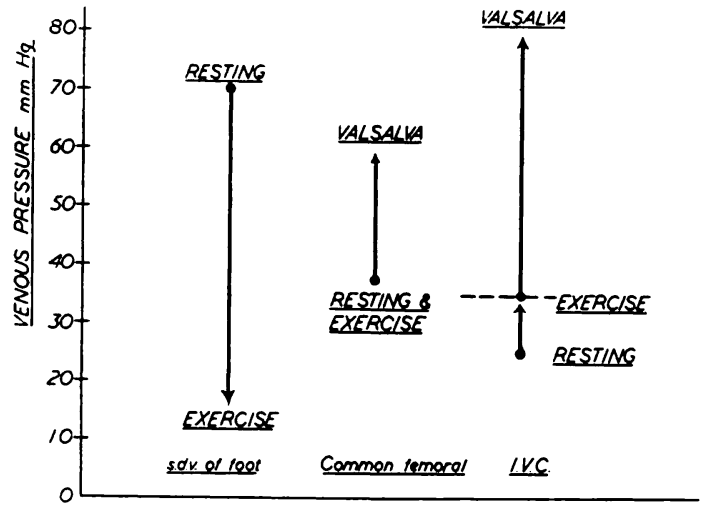

FIG. 8

Diagram to show venous pressure readings, at various levels, on exercise and during the Valsalva tests.
These limited studies suggest that the venous pressure remains virtually stationary at the root of the limb during exercise (Fig. 8). In the inferior vena cava the venous input from the limb exceeds the output, at least during the early period of exercise; consequently the pressure rises, and doubtless aids the venous return to the right side of the heart. The effect of straining and the Valsalva tests are shown on the chart.

\section{VENOUS PRESSURES IN ABNORMAL LIMBS}

The effect of ligation of a major vein It has long been debated whether a normal major vein should be ligated when the accompanying artery is tied, and the following cases seem relevant.

\section{CASE REPORTS}

Case 1-Man aged sixty years. Many years of swelling of the leg with skin changes suggestive of idiopathic elephantiasis. No history of phlebitis. Exercise venous pressures were as follows: standing 90 millimetres $\mathrm{Hg}$.; exercise 30 millimetres $\mathrm{Hg}$. Phlebography showed a normal venous pattern. Ten days after these tests the superficial femoral vein was ligated. Four months later pressure readings were: standing 85 millimetres $\mathrm{Hg}$; ; exercise 80 millimetres $\mathrm{Hg}$. The clinical condition was unaffected by the operation. 
Case 2-Woman aged twenty-five years. Several years' history of idiopathic lymphoedema. Phlebography showed normal venous system. Ligation of superficial femoral vein was undertaken. Four months later venous pressure readings were: standing 77 millimetres $\mathrm{Hg}$; exercise 26 millimetres $\mathrm{Hg}$.

Case 3-Woman aged twenty-two years. Orthostatic swelling below knee for two years. No history of phlebitis. Phlebography revealed normal venous anatomy except for previous ligation of what was described by the operator as a normal popliteal vein. Venous pressure six months after operation were: standing 75 millimetres $\mathrm{Hg}$.; exercise $\mathbf{7 5}$ millimetres $\mathbf{H g}$.

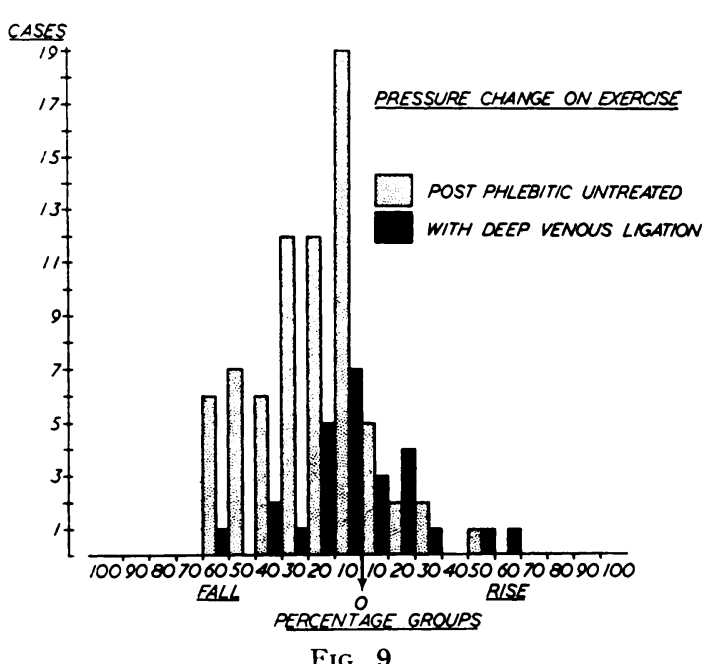

Histogram showing percentage rise or fall of venous pressure on exercise in post-phlebitic limbs untreated, and in those following deep venous ligation. Each column represents the number of cases with percentage pressure changes in the $0-10,10-20$, etc., ranges (percentage groups).
The above case histories indicate that major venous ligation in a limb with normal venous circulation is usually followed by abnormally high venous pressures on exercise. In Case 2 the collateral circulation was adequate to carry the blood flow during exercise, but as years pass the venous circulation will probably become incompetent with consequent pain and swelling.

Venous pressures in chronic deep thrombo-phlebitis-Sevent y-t wo patients have been examined in detail (Fig. 9). The intimate changes in venous pressure readings during muscular contraction and relaxation in the postphlebitic limb are shown in Figure 10. It is significant that in none did the exercise venous pressure fall below 60 per cent of its resting value and in most it remained high and even rose above the resting venous pressure. The degree of abnormality of venous function depends on many factors, notably the site and extent of the original thrombosis, the development of the collateral circulation and the previous venous pressure readings on exercise.

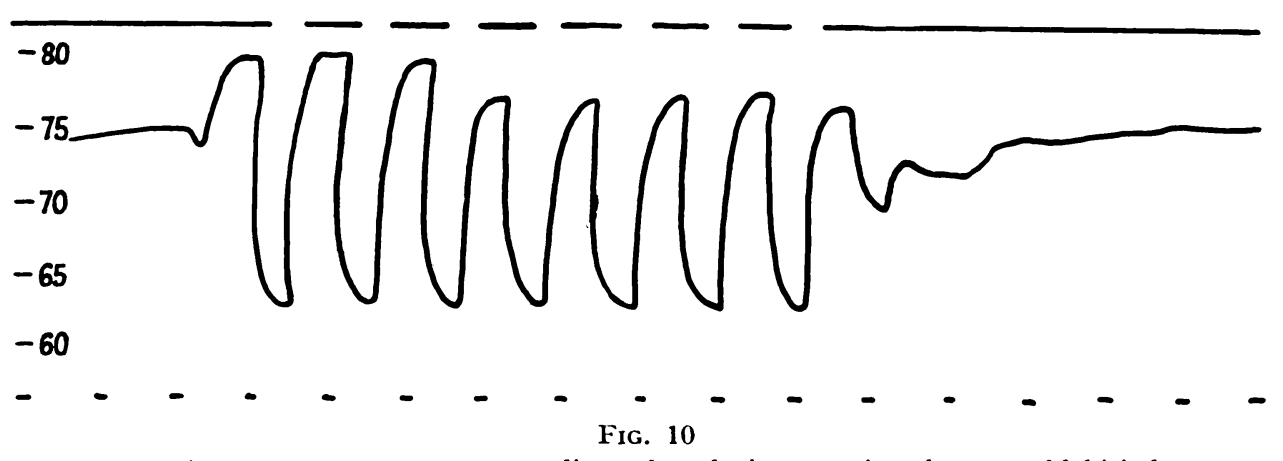

A continuous venous pressure recording taken during exercise of a post-phlebitic leg.

Although we have never failed to demonstrate reopening of the thrombosed vein, either by phlebography or at operation, its extent seems to have no direct relationship to the degree of inefficiency in the return of blood from the lower limb. As will be discussed later in greater detail, we are of the opinion that recanalisation itself is not the principal factor in the post-phlebitic syndrome.

Abnormal pressure readings are constantly found after acute and chronic deep thrombophlebitis, and appear to be the one common etiological factor in the accompanying swelling, 
pain and ulceration. We cannot, however, predict the extent or character of the symptoms from a study of venous pressures alone. One patient with abnormally high exercise venous pressure may have virtually no symptoms, whereas another with similar pressure has ulceration, pain and swelling. The application of a firm lower leg bandage has interesting results. In most subjects the standing pressure rises a few millimetres of mercury and the percentage fall on exercise remains as before the bandaging. If there are grossly incompetent superficial varices, the exercise venous pressure may fall considerably.

In a small number of patients, exercise after the application of a bandage causes the pressure to rise, presumably because of the compression of superficial venous "lakes " which act as a buffer or "sluice-mechanism" during muscular contraction. Such patients find a below-knee bandage uncomfortable.

\section{EXERGISE PRESSURE IN PATIENTS WITH VENOUS INGOMPETENCE}

In those who have superficial varicose veins, the exercise venous pressure falls less than usual (Fig. 11). A normal fall may be obtained by applying a below-knee bandage or by multiple ligation of the incompetent veins. In some patients without superficial varices or
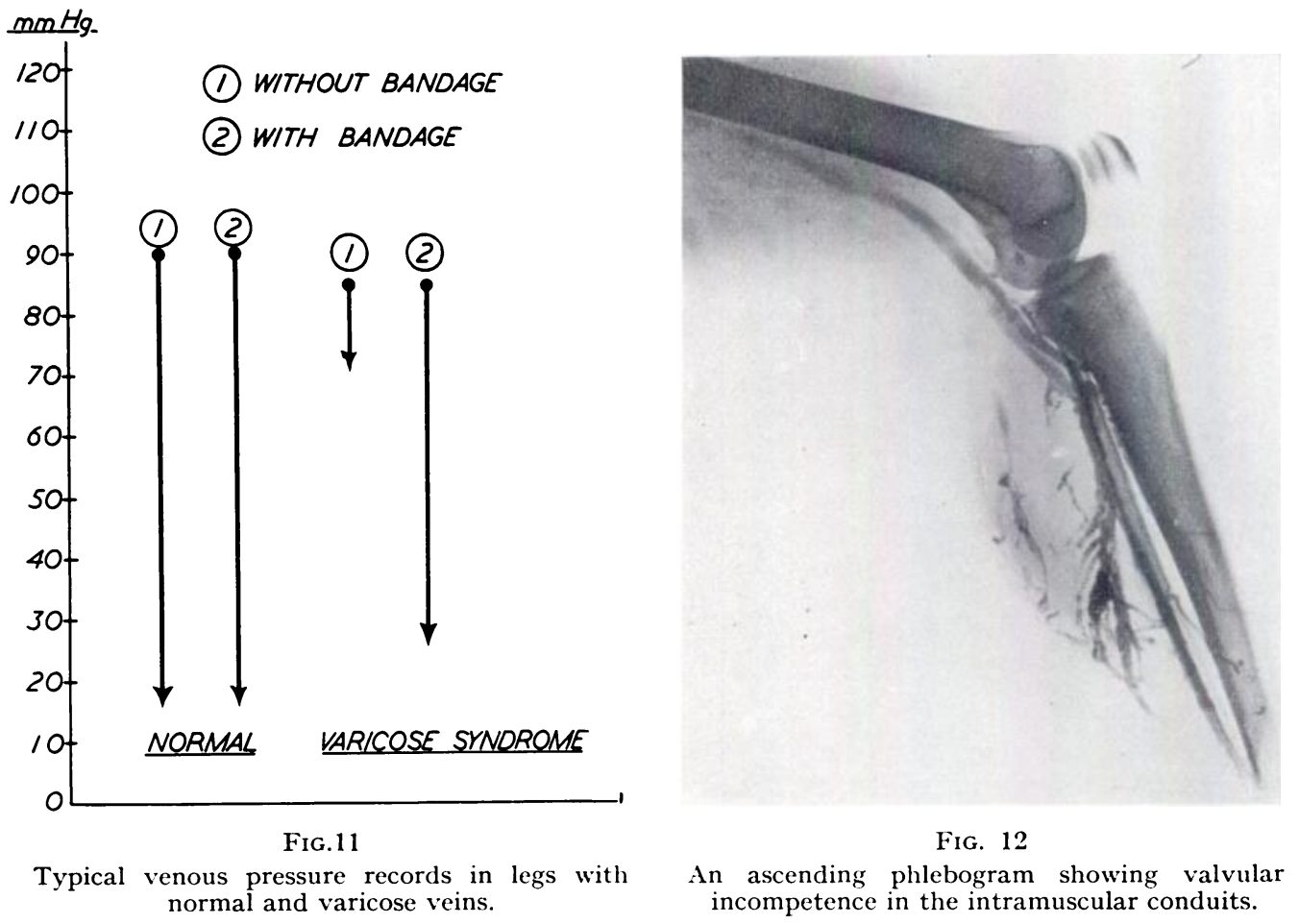

a history of phlebitis, high exercise pressures are found, and phlebography demonstrates varicosities or incompetence in the collecting or intramuscular conduits (Fig. 12). Such patients with deep venous incompetence may suffer pain, swelling and ulceration. If a history of deep venous thrombosis is obtained and marked superficial varicosities are also present, exercise venous pressure with and without a below-knee bandage enables an accurate assessemnt of the separate lesions to be made.

Traumatic ulcers-This type of ulcer is seen in young people who, after a skin abrasion or laceration, acquire a persistent lesion, usually over the lower tibia. Several have been studied and the following explanation is suggested. If the ulcerated and non-ulcerated legs are examined it is found that the exercise venous pressure fails to fall normally and remains in the 0-60 per cent "fall" range. Some patients have marked deep venous incompetence

vol. $34 \mathrm{~B}$, No. 4 , November 1952 
although they are as yet free from symptoms. In normal circumstances this is of no importance, but when a skin lesion over the tibia occurs, continued walking and high exercise venous pressure hinder normal skin healing just as in the limb after phlebitis. The continued low-grade sepsis finally gives rise to periostitis. The syndrome of chronic traumatic ulcer is established. Healing is not difficult to secure if the patient is rested and the ulcer grafted before the stage of periostitis is reached, but after this has occurred great difficulty may be encountered.

The effect of a major venous ligation on the exercise venous pressure-The full assessment of major venous ligation (popliteal or femoral) for the treatment of the limb with chronic deep thrombo-phlebitis will be dealt with in detail in a later paper. Twenty-seven cases have been analysed, some with both pre-operative and post-operative venous pressure readings, but in most only post-operative pressures have been obtained (Figs. 13 and 14). Phlebograms give visual proof that the recanalised vein has been adequately tied. Figure $\mathbf{9}$

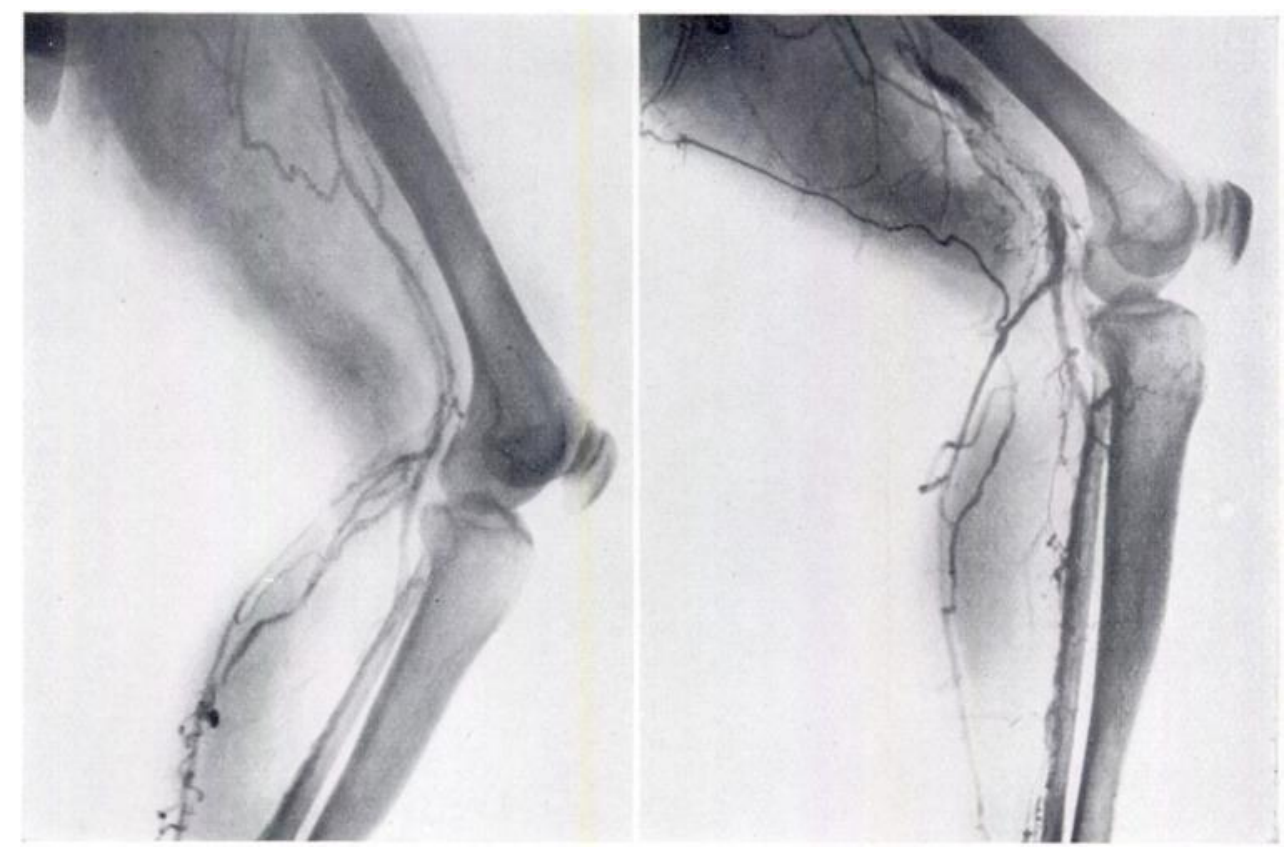
FIG. 13

FIG. 14

Figure 13-Phlebogram of post-phlebitic limb before deep venous ligation. Figure 14-The same leg following deep venous ligation of the recanalised channels.

shows that the range of pressure readings is comparable with, or slightly higher than, those in a similar group of patients in whom the operation has not been performed. When pre-operative readings have been done, the major venous ligation has always caused a decided rise in the post-operative venous pressures. Although some patients claimed improvement after a major venous ligation our assessment of the results as judged by amount of swelling and ulceration was disappointing; the beneficial effects sometimes seen may be better attributed to the rest in bed, the healing of the ulceration, the fitting of an elastic stocking, the correction of anaemia and the education of the patient in care of the limb. Indeed we consider that the operation is based on a faulty conception of the important mechanisms involved in the post-phlebitic limb. Immediately after a major vein thrombosis, gross swelling occurs due mainly to the blockage of the main channel of venous return. The collateral venous circulation develops rapidly and within a month or so of the original episode the limb has greatly decreased in size. Exercise pressures at this stage are high. The peripheral venous 
circulation can sustain for a time such abnormal pressures, and symptoms are minimal. As the years pass the collateral circulation becomes grossly incompetent, the peripheral venous valves give way and the tissues react to the high venous pressures by producing swelling, pain and other symptoms. The major venous thrombosis occurs within a few months of the incident and varies considerably in degree. The more extensive the original thrombosis the worse are likely to be the patient's symptoms because the collateral venous channels are more tortuous and inefficient. If regurgitation down the recanalised deep vein were of major importance it would be supposed that the longer the segment of recanalisation, the greater hindrance it would be to regurgitation and the less the symptoms. In fact the ligation of a recanalised deep vein by decreasing the venous calibre, through which returning blood can be forced, causes a greater obstacle to the muscular pump and increases the venous pressures and incidence of symptoms. This is in agreement with our clinical venous pressure findings.

\section{CONCLUSIONS}

We believe that the technique described is an aid in the analysis of venous abnormalities in limbs. It is simple and safe, and it enables both phlebograms to be made and intravenous pressures to be recorded. Perhaps its greatest use will prove to be in the assessment of therapeutic measures. The protean treatment for leg ulcers illustrates the need for such a criterion.

\section{SUMMARY}

1. A technique for measuring venous pressure during exercise is described.

2. The findings in normal and abnormal conditions of the lower limb are recorded.

3. The mechanisms involved in the return of blood from the lower limb in various conditions are discussed, with particular reference to the post-phlebitic state.

4. The need for an objective method of assessing the results of surgical treatment of venous abnormalities in the lower limb is stressed.

We are grateful to the Department of Medical Photography of the Manchester Royal Infirmary for preparation of the illustrations in this paper.

\section{REFERENCES}

Bedford, D. E., and Wright, S. (1924): Observations on the Venous Pressure in Normal Individuals. Lancet, ii, 106.

Beecher, H. K., Field, M. E., and Krogh, A. (1936): A Method of Measuring Venous Pressure in the Human Leg During Walking. Skandinavisches Archiv für Physiologie, 73, 7.

Clark, J. H., Hooker, D. R., and Weed, L. H. (1934): The Hydrostatic Factor in Venous Pressure Measurements. American Journal of Physiology, 109, 166.

De Camp, P. T., Schramel, R. J., Ray, C. J., Feibleman, N. D., Ward, J. A., and Ochsner, A. (1951): Ambulatory Venous Pressure Determinations in Post-phlebitic and Related Syndromes. Surgery, 29, 44.

Doupe, J., Krynauw, R. A., and SNodgrass, S. R. (1938): Some Factors Influencing Venous Pressure in Man. Journal of Physiology, 92, 383.

Henderson, Y., Oughterson, A. W., Greenberg, L. A., and Searle, C. P. (1936): Muscle Tonus, Intramuscular Pressure and the Venopressor Mechanism. American Journal of Physiology, 114, 261.

Mayerson, H. S., Long, C. H., and Giles, E. J. (1943): Venous Pressures in Patients with Varicose Veins. Surgery, 14, 519.

O'Keeffe, A. F., Warren, R., and Donaldson, G. A. (1951): Venous Circulation in Lower Extremities Following Vein Interruption. Surgery, 29, 267.

McIntire, J. M., and Turner, A. H. (1935): Venous Pressure and Posture in Normal Young Women. Journal of Clinical Investigation, 14, 16.

SmiRk, F. H. (1936): Observations on the Causes of Oedema in Congestive Heart Failure. Clinical Science, 2, 317.

Walker, A. J. (1950): Treatment of Post-phlebitic Leg and Application of Venous-pressure Measurement. British Medical Journal, ii, 1307.

Warren, R., White, E. A., and Belcher, C. D. (1949): Venous Pressures in the Saphenous System in Normal, Varicose and Post-phlebitic Extremities. Surgery, 26, 435.

Vol. $34 \mathrm{~B}$, No. 4, NOVEMBER 1952 